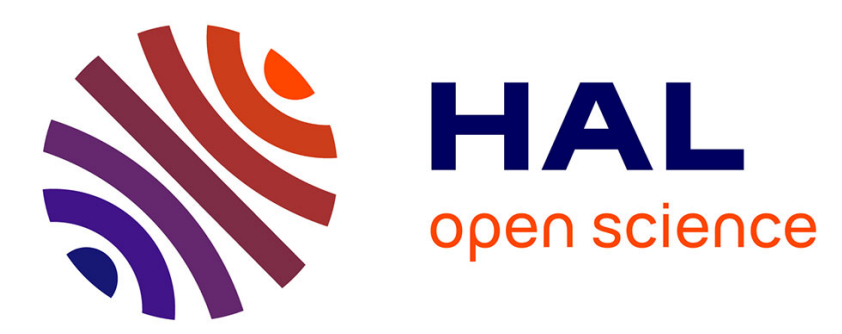

\title{
Fatigue in precipitation hardened materials, a three dimension discrete dislocation dynamics modelling of the early cycles
}

\author{
Chan Sun Shin, Christian Robertson, Marc C. Fivel
}

\section{To cite this version:}

Chan Sun Shin, Christian Robertson, Marc C. Fivel. Fatigue in precipitation hardened materials, a three dimension discrete dislocation dynamics modelling of the early cycles. Philosophical Magazine, 2007, 87 (24), pp.3657-3669. 10.1080/14786430701393159 . hal-00513839

\section{HAL Id: hal-00513839 \\ https://hal.science/hal-00513839}

Submitted on 1 Sep 2010

HAL is a multi-disciplinary open access archive for the deposit and dissemination of scientific research documents, whether they are published or not. The documents may come from teaching and research institutions in France or abroad, or from public or private research centers.
L'archive ouverte pluridisciplinaire HAL, est destinée au dépôt et à la diffusion de documents scientifiques de niveau recherche, publiés ou non, émanant des établissements d'enseignement et de recherche français ou étrangers, des laboratoires publics ou privés. 


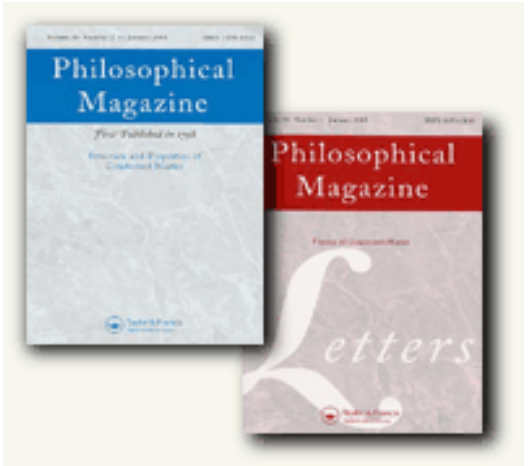

\section{Fatigue in precipitation hardened materials, a three dimension discrete dislocation dynamics modelling of the early cycles}

\begin{tabular}{|c|c|}
\hline Journal: & Philosophical Magazine \& Philosophical Magazine Letters \\
\hline Manuscript ID: & TPHM-07-Jan-0002.R2 \\
\hline Journal Selection: & Philosophical Magazine \\
\hline $\begin{array}{r}\text { Date Submitted by the } \\
\text { Author: }\end{array}$ & 10-Apr-2007 \\
\hline Complete List of Authors: & $\begin{array}{l}\text { SHIN, Chan; KAERI } \\
\text { robertson, christian; CEA/Saclay, DMN } \\
\text { Fivel, Marc; SIMAP-GPM2, CNRS/INPG }\end{array}$ \\
\hline Keywords: & dislocation dynamics, fatigue \\
\hline Keywords (user supplied): & precipitate \\
\hline
\end{tabular}

\section{S ScholaroNE \\ Manuscript Central}




\title{
Fatigue in precipitation hardened materials, a three dimension discrete dislocation dynamics modelling of the early cycles
}

\author{
C.S. SHIN $\dagger$, C.F. ROBERTSON $\ddagger$ and M.C. FIVEL*§ \\ $\dagger$ Korea Atomic Energy Research Institute, Daejeon, 305-353 Korea \\ † CEA/SRMA Saclay, building-455, 91191 Gif-sur-Yvette, France \\ § SIMAP, CNRS/INPG, BP 46, Domaine Universitaire, 38402 St Martin d'Hères, France \\ *Corresponding author Email : Marc.Fivel@inpg.fr \\ Abstract
}

Three-dimensional dislocation dynamics (DD) fatigue simulations in precipitation hardened metals is a major challenge in terms of numerical development. Several precipitate configurations have been investigated with an original treatment of precipitate-dislocation interactions and a parallelized DD code. In grains containing single-sized shearable particles $\left(r_{p}=160 \mathrm{~nm}\right)$, strain is localized in clear bands where the precipitates are totally sheared-off. The fatigue behaviour involves an initial hardening followed by severe cyclic softening and significant surface slip irreversibility. In the presence of large single-sized particles $\left(r_{p}=400\right.$ $\mathrm{nm})$, the PSB structures is accompanied by highly reversible surface displacements. Slip dispersion originates from Orowan loops that have little effect on the mechanical response. The mechanical behaviour of a bimodal distribution is intermediate between the two above cases with the above micro-structural features coexisting within the same grain. Unlike in the mono-modal large particle case where all the particles retain their initial strength, some of the large particles of the bimodal distribution undergo a significant strength reduction.

Keywords: Dislocation dynamics; Fatigue; Precipitates 


\section{Introduction}

In the past, precipitation hardening of ductile metals has been examined in details, both experimentally [1, 2] and theoretically [3, 4]. Early numerical models treated isolated dislocation lines gliding in a single slip plane. These models were developed with a view to examine the effect of strong and weak point obstacles on the flow stress [5]. More recently, numerical models treating distributions of finite size particles have been worked out; accounting for the particle shape effect [6] and the stress field due to the particle/matrix elastic constant mismatch [7, 8]. Dislocation/particle interactions were also investigated using three-dimensional dislocation dynamics modelling, in monotonic loading conditions $[9,10]$.

At the same time, the effect of cyclic loading has been examined in DD in a single-phased metal. These simulations provided information regarding dislocation microstructure formation, stress-strain behaviour [11], persistent slip marking growth and micro-crack nucleation [12]. The present work represents a preliminary attempt to treat the case of fatigue in precipitation hardened metals, combining information from these prior investigations. This case is a major challenge in terms of numerical development, since the behaviour of a large population of dislocations and their interactions with a large number of precipitates need to be treated. Hence, a parallel DD code has been developed, together with an original method accounting for the possibility of precipitate shearing.

This numerical approach, described in section 2, is used to investigate deformation in precipitation hardened grains comprising either small (section 3.1), large (section 3.2), or a combination of the two types of precipitates (section 3.3). 


\section{DD model overview}

\subsection{Fatigue modelling}

In DD models, the dislocation lines are treated as line singularities embedded in an elastic medium. In the DD model used here, the line singularities are discreticized into pure screw and edge segments gliding in a rigid ${ }^{1}$ face centred cubic (fcc) crystal lattice, with a lattice parameter of 10 times the Burgers vector magnitude. The velocity of a given dislocation segment is simply the effective resolved shear stress times a drag coefficient modelling the dislocation/phonon interaction. The materials parameters used in the model characterize a low stacking fault energy metal similar to austenitic steels. The screw character segments can change their slip planes by cross-slip, using the stochastic procedure as described in [13]. Hence, a cross slip probability $P$ over each time step is first computed using the expression:

$$
P=\beta \frac{l}{L o} \frac{\delta t}{t o} \exp \left(\frac{\tau_{d}-\tau_{I I I}}{k T} V\right)
$$

where $\beta$ is a normalisation coefficient ensuring that $(0<\mathrm{P}<1), l$ is the length of the considered screw segment, $L_{0}, t_{0}$ are respectively a reference length and time, $\delta t$ the discrete time step, $V$ the activation volume, $\tau_{d}$ the effective resolved shear stress in the cross slip system and $\tau_{\text {III }}$ a threshold stress. A random number $\mathrm{N}$ between 0 and 1 is first generated. Dislocation cross slip occurs for screw segments only if $\mathrm{N}<P$. In the following simulations, the parameters used for cross-slip at $300 \mathrm{~K}$ are $L_{0}=1 \mu \mathrm{m}, t_{0}=1 \mathrm{~s}, \tau_{\mathrm{III}}=51 \mathrm{MPa}$ and $\mathrm{V}=$ $2117 b^{3}$

\footnotetext{
${ }^{1}$ No lattice rotations are considered.
} 
The fatigue simulation boundary conditions are exactly the same as described in [11]: cylindrical grain geometry is considered for the shape of the simulated space with a fixed diameter of $10 \mu \mathrm{m}$. Surface grains are represented by appropriate properties of the boundaries. On the top surface, the dislocations are free to escape whereas all other surfaces act as strong obstacles to dislocation motion simulating high disordered grain boundaries. The escaping dislocations print surface steps that are computed using a specific post-treatment method. For the grain orientation and size used here, the image forces induced by the free surface have practically no effect on the dislocation structure and surface relief, as shown in details in [12]. Therefore, the image-forces are neglected in this study.

The initial dislocation microstructure consists of a single pinned dislocation segment. Indeed, it was demonstrated in [11-12] that such a simple initial configuration leads to realistic cyclic behaviour. During the first quarter of cycle (i.e. the first loading stage), dislocation density strongly increases so that as soon as the first unloading stage, there is a sufficient mobile dislocation density to accommodate the imposed plastic strain.

The calculations are performed under isothermal conditions at $300 \mathrm{~K}$, using an homogeneous applied stress tensor selected for single slip. The applied stress level is stepwise increased, with a typical stress step increment of $1 \mathrm{MPa}$. Each time the load is increased, the dislocations move until the whole dislocation microstructure is in equilibrium with the external loading and satisfies the quasi-static condition [11]. The imposed plastic strain range condition is achieved using a feedback control loop based on the cumulated plastic strain. This quantity is computed at each time step, from the dislocation glide summed up over all the active slip systems. Throughout the cycles, the applied load is reversed each time that the total slip is equal to the pre-selected maximum (or minimum) plastic strain level. In this study, the maximum plastic strain level was fixed to $\pm 5 \times 10^{-4}$, corresponding to the imposed plastic strain range $\Delta \varepsilon_{\mathrm{p}}=10^{-3}$. 
(i)- the elastic deformation of the precipitates,

(ii)- the precipitate barrier effect (as for highly disordered grain boundary),

(iii)- the progressive loss of the precipitate strength, after repeated shear-off,

(iv)- the specific lattice friction acting on the dislocation within the precipitates.

\section{[Insert figure 1 about here]}

The effect (i) comes from the elastic constant mismatch between the precipitates and the matrix, generating image-stresses in the vicinity of the precipitates when a dislocation approaches the particle [8]. In a detailed preliminary study [9], it was shown that the effect (i) has a limited influence on the yield stress (less than $+4 \%$ ) and the strain hardening characteristics of the crystal (less than $+10 \%$ ), especially when cross-slip is accounted for. In the massive simulations to be presented, the image forces due to the precipitates are thus neglected, in order to reduce the overall computational load.

The effect (ii) is due to the lattice parameter misfit between the matrix and precipitate. This phenomenon is modelled by the implementation of internal barriers to dislocation motion called "facets", placed inside of the simulation volumes (see Fig.-1a). These barriers or facets can be sheared by the moving dislocations, provided a locally defined stress criterion is satisfied. In the simulations, each precipitate is described by interconnected facets, forming 
closed volumes. More specifically, the precipitates are made of two 6 faced pyramids, attached together by their basal planes (Figure 1b).

The effect (iii) is modelled by reducing the critical crossing stress of a given facet [14]. Hence, the individual facet strength is decreased at the time of each dislocation passage, until a critical minimum value is attained. Beyond that point, the facet strength is set to zero, as shown in Figure-1c. Such behaviour represents a first order approximation of the precipitate dissolution in the matrix, a process driven by the surface/volume ratio of the sheared-off precipitates.

The effect (iv) can significantly affect the dislocation line curvature, inside the precipitate. This phenomenon is here neglected, in consideration of the small particle sizes as compared to the individual dislocation lines. The effect (iv) is also neglected in simulations performed with large precipitates (see section-3.1), due to their high shear resistance.

The development of a new parallel DD code is regarded as an essential step, towards the achievement of fatigue simulations including a large number of precipitates [15]. The parallelisation scheme adopted here is based on a space decomposition of the simulation volume into smaller entities called boxes. Box subsets treating both dislocations and facets are then attributed to individual CPUs. The computational load balance between the various processors is performed using a method accounting for the current dislocation arrangements. When the dislocation density is rather uniform in the simulation volume, a speed up factor of 17 is obtained using 25 CPUs handling more than 30,000 segments [16]. In the fatigue simulations however, the dislocation density is highly heterogeneous due to strain localization into persistent slip bands, yielding a lower computational efficiency. 


\subsection{Simulation cases}

The number of facets that can be treated using the available simulation settings is limited by the RAM memory restrictions imposed by the new parallel algorithm [16]. Hence, a typical grain of 10 micrometers diameter containing exactly the same precipitate sizes and densities as in actual superaloys remains beyond the capacities of this new DD code, using the available computing machines. Therefore no direct comparisons with TEM observation data is presented in this paper, for direct model validation. This could possibly be done in the future, as soon as improved hardware and software developments will become available.

Three different particle distributions have been investigated.

(i) A monomodal distribution of $14 \%$ volume fraction of large $\left(r_{p}=400 \mathrm{~nm}\right)$ particles.

(ii) A monomodal distribution of $14 \%$ volume fraction of small $\left(\mathrm{r}_{\mathrm{p}}=160 \mathrm{~nm}\right)$ small particles.

(iii) A bi-modal distribution of $14 \%$ volume fraction of small and large particles, i.e. $7 \%$ of small precipitates and $7 \%$ of large precipitates.

For each simulated case, the results are presented in terms of stress amplitude, dislocation microstructure and surface slip marking evolutions.

\section{Results}

\subsection{Case-1: the mono-modal distribution of large particles}


In this section, the simulation volume includes a total of 161 precipitates with a radius $r_{p}=400 \mathrm{~nm}$, yielding a precipitate volume fraction of $14 \%$. Here, the initial facet strength is set to $7.3 \mathrm{GPa}$ and the ultimate facet strength is set to $162 \mathrm{MPa}$ [14]. This implies that the particles are practically non-shearable. The total number of cycles performed is $\mathrm{N}_{\max }=5$ cycles. As mentioned before (in section-2), the simulations are performed in imposed plastic strain range conditions. The corresponding stress amplitude and dislocation density evolutions are shown in figure-2, where they are compared to those obtained in a particle-free simulation volume of exactly the same orientation and size as in the present case. The overall effect of the precipitates on the mechanical response and dislocation densities is limited, whereas a major effect on the dislocation structures is obtained.

\section{[Insert figure 2 about here]}

During cycle-1: the initial dislocation sources are activated and shear loops are emitted one after the other, until pile-ups are formed at the grain boundaries. The precipitates are bypassed by the moving dislocations thanks to the Orowan mechanism, whereby one loop is left around each by-passed precipitate. Then, these "Orowan loops" accumulate in the neighbourhood of each by-passed precipitates, in their original glide plane.

During cycles 2-5: when the critical cross-slip stress is attained, the (screw-type) dislocations positioned around the precipitates ${ }^{2}$ undergo a glide plane change towards the cross-slip plane. This process generates high density, immobile dislocation tangles around the precipitates. Later on, the tangles can act as pinning point for the mobile dislocations gliding between the precipitates. These interactions contribute to increase the dislocation density in the pre-

\footnotetext{
${ }^{2}$ Either in Orowan loops or in incoming dislocations.
} 
In the surface, the initial slip markings are made of highly reversible steps, approximately as long as the grain diameter. These slip steps (see Fig. 3c) are progressively transformed into shorter, ribbon-like markings (see Fig. 3d), while the inter-precipitate dislocation density progressively increases. At the end of the simulated time, the ribbon lengths are comparable to the average inter-precipitate distance. The surface displacements remained fully reversible during the whole of the simulated time. In single phase, particle-free simulations [11] it was found that reversible surface slip is generally due to simple shear loading conditions associated with few pinned dislocations (or sources). Irreversible surface slip is due to the channel dislocations (prismatic loops), obtained from prior dipolar sub-structures. In the mono-modal particle case, the PSB structures are devoid of channels or dipolar structures, yielding reversible surface displacements.

[Insert figure 3 about here]

\subsection{Case-2: the mono-modal distribution of small particles}


3.2.1 DD simulation results. In this case, the simulated volume contains 2510 small particles with $r_{p}=160 \mathrm{~nm}$, again yielding a volume fraction of $14 \%$. The initial critical particle shearing stress is $292 \mathrm{MPa}$, which is decreased each time the particle is sheared-off by an incoming dislocation. The ultimate critical shear stress attributed to a given particle is $160 \mathrm{MPa}$. These characteristics simulate the effect of particle dissolution or phase change, after repeated shearing. The total number of cycles performed in this case is $\mathrm{N}_{\max }=3$ cycles, while the dislocation microstructure progressively forms, during the following steps.

During cycle-1: the initial sources are activated and shearing loops are emitted in their respective, initial glide planes. The precipitates crossing the active planes are sheared-off by the incoming dislocations and pile-ups are formed at the grain boundaries. Dislocation accumulation in the pile-ups ceases as soon as the critical cross-slip stress threshold is attained. Unlike in precipitate-free materials, the presence of the large precipitates outside of the initial slip planes strongly restricts the gliding distance of the cross-slipped dislocations. Hence, stress accumulates much faster in the cross-slip system and so, the dislocations tend to cross-slip back into (parallel) primary slip planes positioned very close to the initial ones. In precipitate-free grains, dislocation loops tend to glide back and collapse as the applied load decreases, due to the line tension stress. Here, the collapsing process in a given glide plane is impeded (to some extent) by the particles, yielding numerous residual dislocations and reduced slip reversibility. Hence, the dislocation density increase during the first applied cycle is very fast (see Fig. 2a), since: i- a sizable fraction of the dislocations accumulates in their initial glide plane as residual dislocations, ii- numerous new dislocation sources have to be formed (thanks to double cross-slip), in order to keep on accommodating the imposed plastic strain. 
[Insert figure 4 about here]

The precipitates crossing the interfacial dislocation pile-ups planes are repeatedly sheared-off, until their mechanical resistance completely vanishes. This phenomenon is shown in figure-5b, where the colour ascribed to each particle refers to its residual mechanical strength. Statistically, only $1 \%$ of the precipitates exhibit a significant decrease of their critical shear stress (see Fig. 5a). The data thus reveals a very pronounced degree of strain localisation ${ }^{3}$, also characterized by a relatively quick cyclic softening (see Fig. 2b). At the free surface, the initial surface slip markings are made of reversible steps approximately as long as the grain diameter (see Fig. 4c). These slip steps are soon replaced by tongue-like extrusions (or intrusions). The tongue-like surface markings are associated with the channel dislocations

\footnotetext{
${ }^{3}$ Sometimes called "dislocation channeling".
} 
(prismatic loops) located inside of the grain (see Fig. 4d). The extrusions (intrusions) grow with the number of cycles, revealing significant surface slip irreversibility. The reversible slip markings are located at the interface between the PSB and the matrix and thus, are associated with the above described "mobile interfacial dislocations". Qualitatively, the DD simulations in this section yield the main dislocation features as obtained in fatigue in industrial alloys containing small precipitates, i.e.: i- PSB structures and ii-their associated, precipitate-free clear bands (see [17-20] in Inconel-718, for example).

[Insert figure 5 about here]

\subsection{Case-3: the bi-modal distribution of small and large particles}

3.3.1 DD simulation results. Alloys presenting a bimodal size particle distribution combine good strength (merit of the small particles) and good cyclic stability (merit of the large particles). In this section, the simulation volumes comprise a bi-modal distribution of 1456 small particles with $r_{p}=160 \mathrm{~nm}$ and 57 large particles with $r_{p}=400 \mathrm{~nm}$. The total number of simulated cycles is $\mathrm{N}_{\max }=4$ cycles. The addition of large particles decreases the degree of cyclic softening seen in the mono-modal case with small particles $\left(r_{p}=160 \mathrm{~nm}\right)$. In fact, the mechanical response in this case lies between the two previously investigated, mono-modal cases (see Fig.2b). The total dislocation densities and the rate of dislocation accumulation decreases with the percentage of large particles (see Fig.2a). In the grain, the micro-structural characteristics of the small and large particle mono-modal cases coexist. Typical features of the small particle mono-modal case are clearly visible within the interspaces between the large precipitates (see Fig. 6a). These sub-structures include channels, mobile dislocations and tangles (see Fig. 6b). Parts of the tangles are made of the residual loops left around the nonshearable particles, due to the Orowan mechanism. The mobile dislocations and their 


\section{[Insert figure 6 about here]}

In this case, unlike in the mono-modal case described in section 3.2, the small sheared-off particles are scattered across the whole grain (see Fig. 7b). Hence, strain localisation is far less pronounced than in section 3.2. This effect is consistent with the statistical data presented in Fig. 7a, showing that a sizable fraction (larger than 1\%) of the particles undergo a significant strength reduction (compare Fig. 7a to Fig. 5a). In addition, unlike in the monomodal particle case $\left(r_{p}=400 \mathrm{~nm}\right)$, where all the large particles keep their initial strength, it is found that some of the large particles undergo a significant strength reduction. This effect is ascribed to the dislocation substructures (channels and mobile dislocations) forming between the large precipitates. These substructures generate sufficient local strain to occasionally shear the large particles.

[Insert figure 7 about here] 
Qualitatively, the simulated dislocation structures are quite comparable with those found in industrial alloys containing large particle (e.g. Waspaloy), where high density dislocation tangles are clearly visible in the vicinity of the large precipitates (see [20-22], for example).

\begin{abstract}
4 Summary
3D dislocation dynamics simulations in fatigue have been performed in $10 \mu \mathrm{m}$ cylindrical simulation volumes, containing three types of particle distributions: i- mono-modal, small sized particles, ii- mono-modal, large sized particles and iii- a bi-modal particle distribution. In the simulations, the applied loading is symmetrical traction-compression oriented for single glide using an imposed plastic strain range of $\Delta \varepsilon_{\mathrm{p}}=10^{-3}$. The available computational power imposes limitations on the practical volume fraction treated in the simulations. Hence, the fixed particle volume fraction of $14 \%$ is used here. In spite of these limitations, the first simulation results presented here provide valuable insight regarding the dislocation microstructure formation and the stress-strain behaviour observed in fatigued super-alloys. From the DD simulation results, it is shown that:
\end{abstract}

1- In grains having a mono-modal distribution of large particles, the dislocation density is similar to single phased materials: the associated surface displacements are ribbon-like and highly reversible. The presence of these particles induces a small effect on the cyclic mechanical response when compared to the precipitate free material.

2- In grains having a mono-modal distribution of small particles, strain localises into PSB structures with features similar to single phased materials, in spite of their 
higher dislocation density and their accompanying clear bands. A large effect is observed in the initial stress amplitude, followed by severe cyclic softening.

3- In grains having a bi-modal particle distribution, the obtained results lie between case-1 and case-2, as regards: the stress-strain behaviour, the PSB microstructural characteristics, the associated surface slip marking geometry and reversibility, the dislocation densities, the degree of strain-localisation and the cyclic mechanical response. Unlike for case 1 where all the large particles keep their initial strength, it is found that some of the large particles are sporadically sheared.

\section{At this point, more work is needed in order to achieve further validation steps. In particular, it} is suitable to precisely compare the irreversible slip fraction, the corresponding extrusion (intrusion) growth rate with experimental data now available thanks to the AFM technique. This could be accomplished through simulations of a larger number of cycles, at least up to the point of saturation of both the dislocation microstructure and the mechanical behaviour. Moreover, quantitative comparisons with industrials materials require the real precipitate size to be accounted for. As an example, Waspaloy contains 20 percent of a bimodal distribution of 80 nanometers and 15 nanometers particules which is about ten times smaller than in the case 3 presented here. Thus, a direct comparison needs a numerical tool capable to treat a significantly larger number of particles (factor 1000). 


\section{References}

[1] R. Ebeling and M.F. Ashby, Phil. Mag. 13805 (1966).

[2] F.J. Humphreys and J.W. Martin, Phil. Mag. 16927 (1967).

[3] A. Kelly and R.B. Nicholson, Strengthening Method in Crystals, (New York: Wiley, p. 156 1971).

[4] B. Reppich, Mater. Sci. Tech. 6311 (1993).

[5] A.J.E. Foreman and M.J. Makin, Phil. Mag. 151011 (1966).

[6] A.W. Zhu, and E. A. Starke Jr, Acta Mater. 473263 (1999).

[7] V. Mohles and E. Nembach, Acta Mater. 492405 (2001).

[8] C. Shin, M.C. Fivel and K.H. Oh, J. Phys. IV 11 Pr5 (2001).

[9] C.S. Shin, M. C. Fivel, M.Verdier and K. H. Oh, Phil Mag. A 833691 (2003).

[10] C.S. Shin, M.C. Fivel and W W Kim, Modelling Simul. Mater. Sci. Eng. 131163 (2005).

[11] C. Déprés, C.F. Robertson and M.C. Fivel, Phil. Mag. A 842257 (2004).

[12] C. Déprés, C.F. Robertson and M.C. Fivel, Phil. Mag. A 8679 (2006).

[13] M. Verdier, M.C. Fivel and I. Groma, Modelling Simul. Mater. Sci. Eng. 6755 (1998).

[14] R.E. Stolz and A. Pineau, Mat. Sci. Eng. 34275 (1978).

[15] C. Shin, Doctoral thesis, laboratory GPM2, INP Grenoble, (2004).

[16] C.S. Shin, M.C. Fivel, M. Verdier and S.C. Kwon, J. Comp. Phys., 215(2), pp. 417-429 (2006).

[17] F. Alexandre, Doctoral Thesis, Ecole des Mines de Paris (2004)

[18] V. Brien, B. Décamps, Mater. Sci. Eng., A316, pp. 18-31, (2001).

[19] M. Clavel, A. Pineau, Mat. Sci. Eng. 55157 (1982).

[20] C. Robertson, S. Deyber, F. Alexandre, unpublished work.

[21] H.J. Penkalla, J. Wosik, A. Czyrska-Filemonowicz, Mater. Chem. Phys., 81, pp.417-423, (2003).

[22] J. Wosik, B. Dubiel, A. Kruk, H-J. Penkalla, F. Schubert, A. Czyrska-Filemonowicz, Mater. Char., 46, pp.119-123, (2001). 


\section{FIGURE CAPTIONS}

Figure-1. Numerical treatment of dislocation/precipitate interactions.

(a) Schematic representation of one isolate internal interface called facet,

(b) typical shape for a precipitate defined by an assembly of six facets,

(c) evolution of the facet strength as a function of the number of dislocation passages.

Figure-2. Evolutions with the number of cycles obtained using various simulation cases. (a) Evolution of the dislocation densities, (b) evolution of the stress amplitude. The bi-modal case curve is not shown for clarity.

Figure-3. Results obtained in a simulation volume containing a mono-modal distribution of un-shearable particles. (a) Three dimensional representation of the dislocation microstructure after $\mathrm{N}=5$ cycles viewed from a direction parallel to the primary slip plane, (b) persistent slip band microstructure after $\mathrm{N}=5$ cycles, (c) surface slip markings after $\mathrm{N}=1$ cycle: long ribbons, (d) surface slip markings after $\mathrm{N}=5$ cycles: shortened ribbons.

Figure-4. Results obtained in a simulation volume containing a mono-modal distribution of small particles. (a) Persistent slip band dislocation microstructure after $\mathrm{N}=3$ cycles, (b) threedimensional view of the dislocation microstructure after $\mathrm{N}=3$ cycles, (c) surface displacements associated with a persistent slip band, after $\mathrm{N}=1$ cycle, (d) after $\mathrm{N}=3$ cycles.

Figure-5. Results obtained in a simulation volume containing a mono-modal distribution of small particles. (a) Mapping of the residual mechanical strength of the particles, superimposed on the dislocation microstructure, (b) statistical analysis of the residual mechanical strength of the particles, 
Figure-6. Results obtained in a simulation volume containing a bi-modal distribution of small and large particles. (a) Persistent slip band dislocation microstructure after $\mathrm{N}=4$ cycles, (b) three-dimensional view of the dislocation microstructure after $\mathrm{N}=4$ cycles, (c) surface displacements associated with a persistent slip band after $\mathrm{N}=1$ cycle, (d) after $\mathrm{N}=4$ cycles.

Figure-7. Results obtained in a simulation volume containing a bi-modal particle distribution. (a) statistical analysis of the residual mechanical strength of the particles, (b) mapping of the residual mechanical strength of the particles, superimposed on the dislocation microstructure. 


\section{Figure-1}

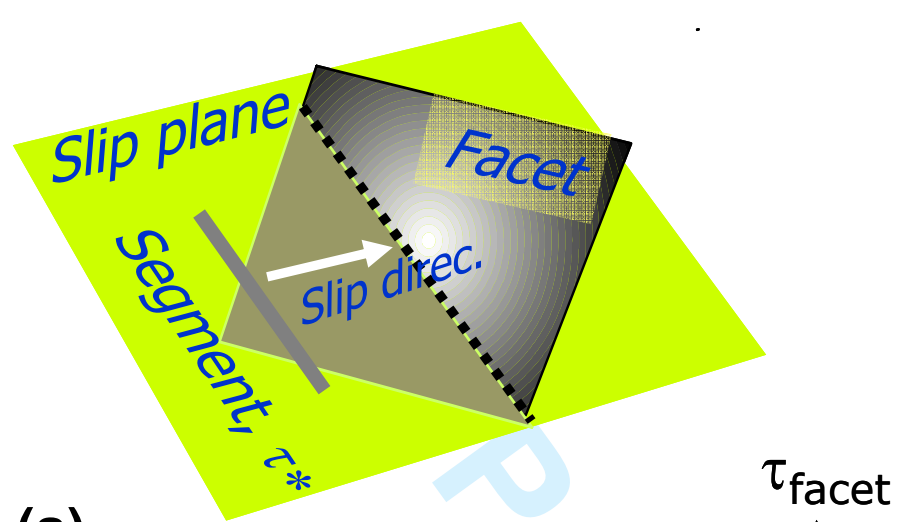

(a)

(b)
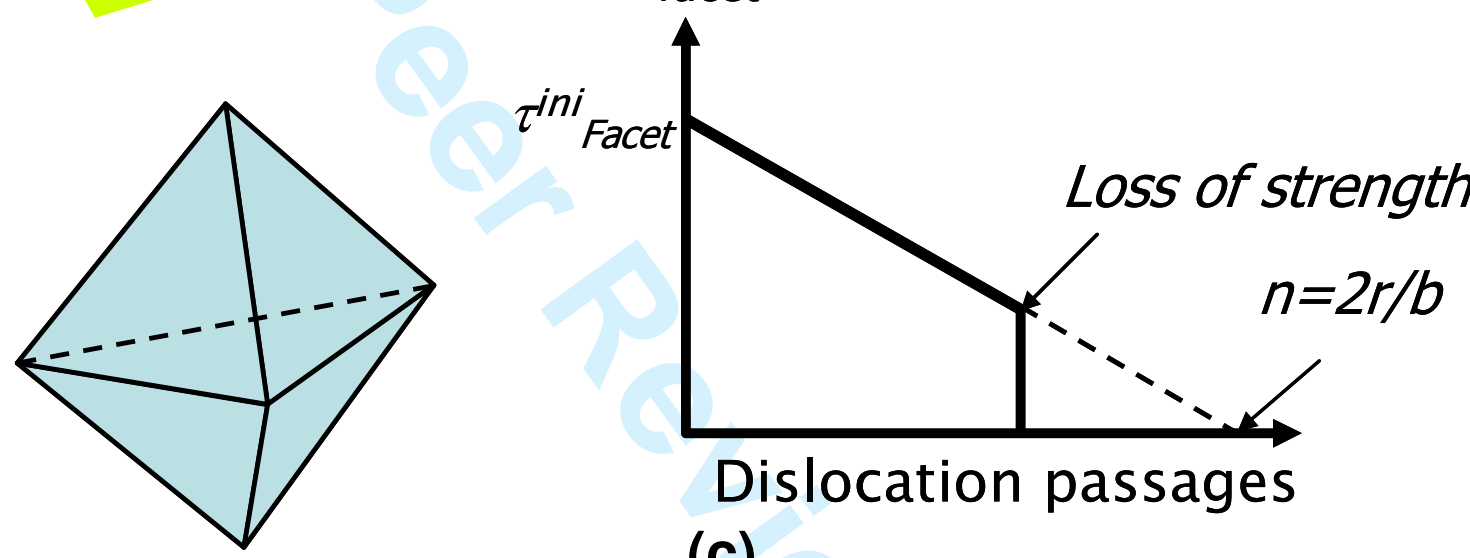

(c)

Figure-1. Numerical treatment of dislocation/precipitate interactions.

(a) Schematic representation of one isolate internal interface called facet,

(b) typical shape for a precipitate defined by an assembly of six facets,

(c) evolution of the facet strength as a function of the number of dislocation passages. 


\section{Figure-2}

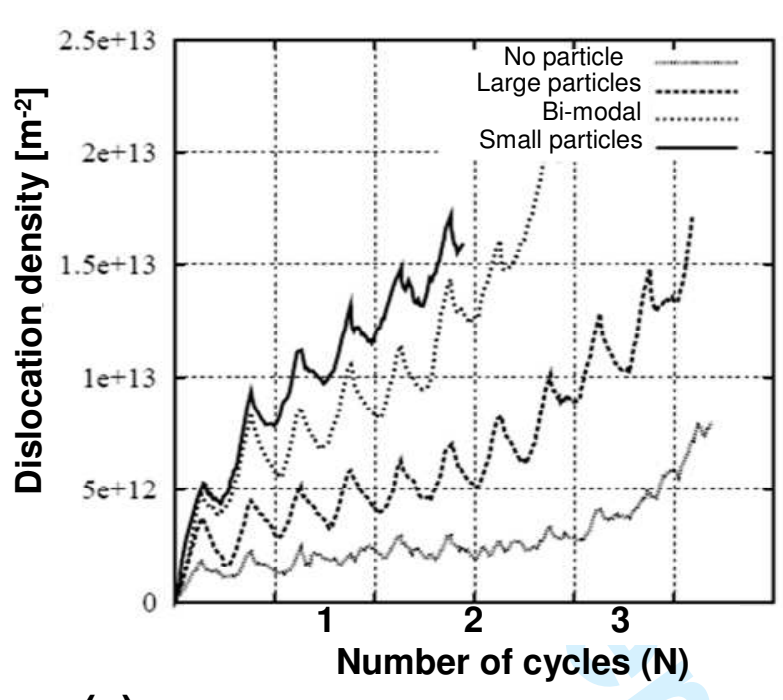

(a)

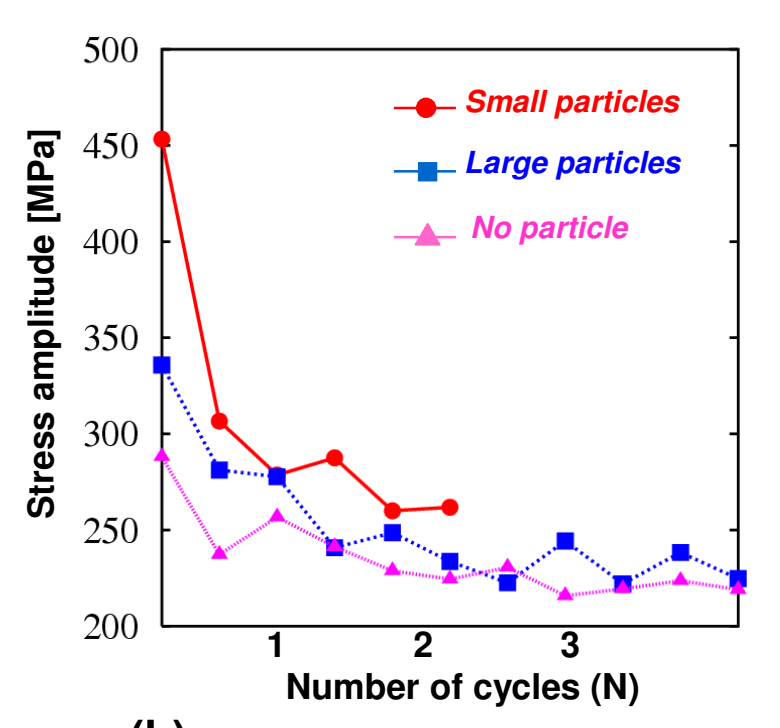

(b)

Figure-2. Evolutions with the number of cycles obtained using various simulation cases.

(a) Evolution of the dislocation densities, (b) evolution of the stress amplitude. The bi-modal case curve is not shown for clarity. 


\section{$\underline{\text { Figure-3 }}$}

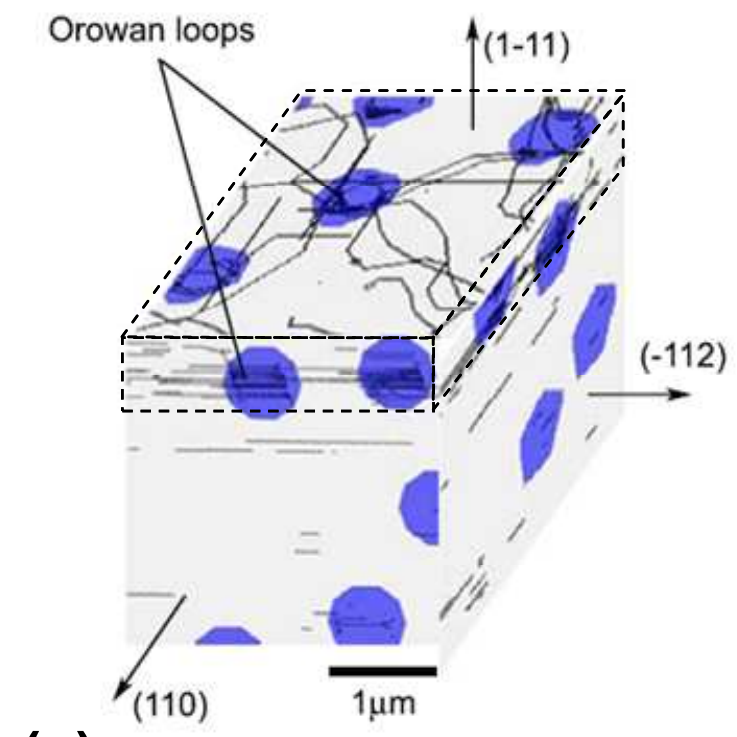

(a)

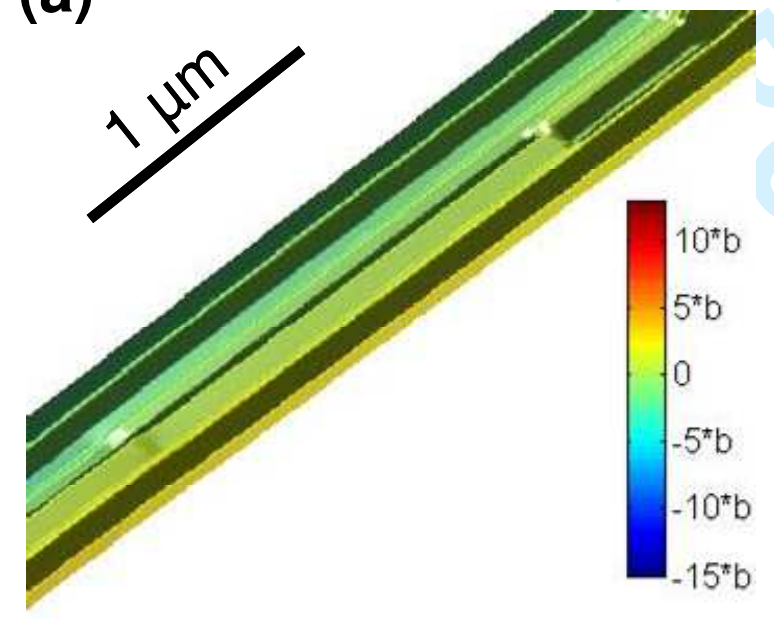

(c)

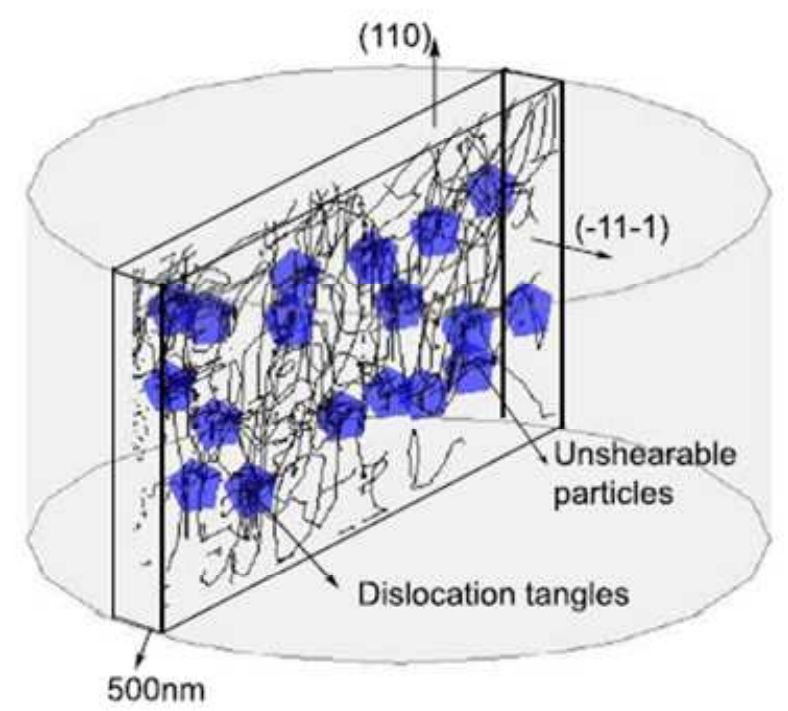

(b)

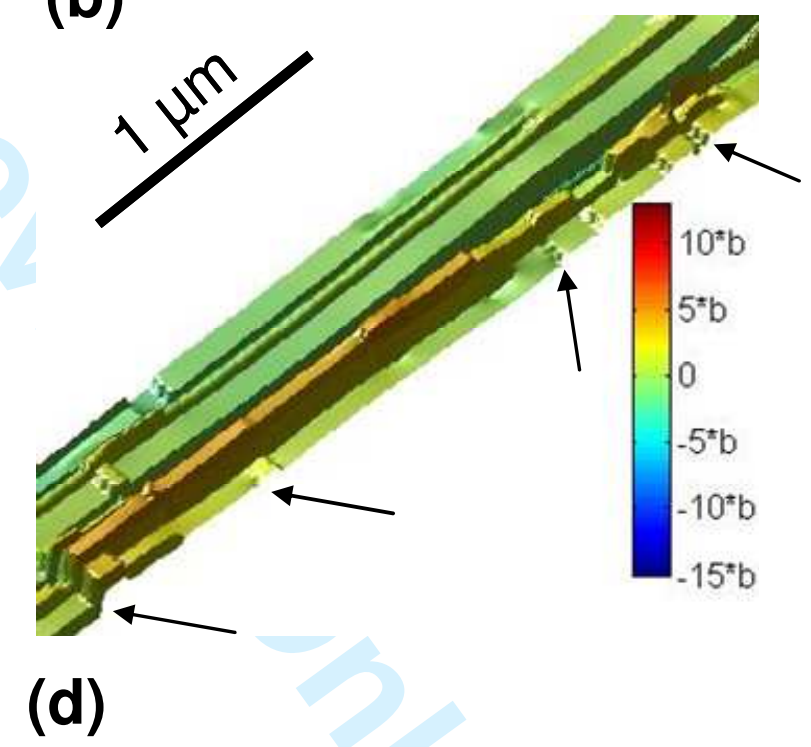

Figure-3. Results obtained in a simulation volume containing a mono-modal distribution of un-shearable particles. (a) Three dimensional representation of the dislocation microstructure after $\mathrm{N}=5$ cycles viewed from a direction parallel to the primary slip plane, (b) persistent slip band microstructure after $\mathrm{N}=5$ cycles, (c) surface slip markings after $\mathrm{N}=1$ cycle: long ribbons, (d) surface slip markings after $\mathrm{N}=5$ cycles: shortened ribbons (see arrow markers). 


\section{Figure-4}
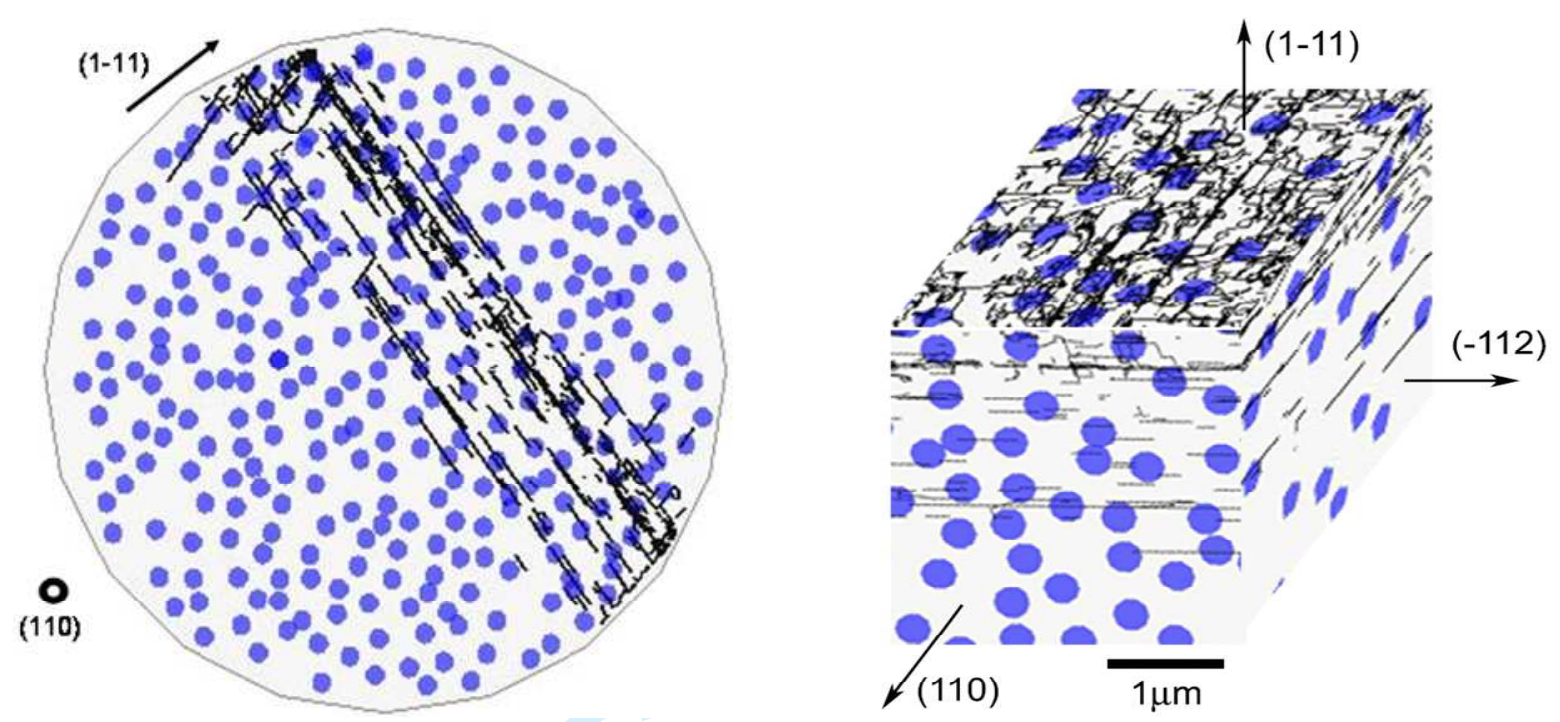

(a)

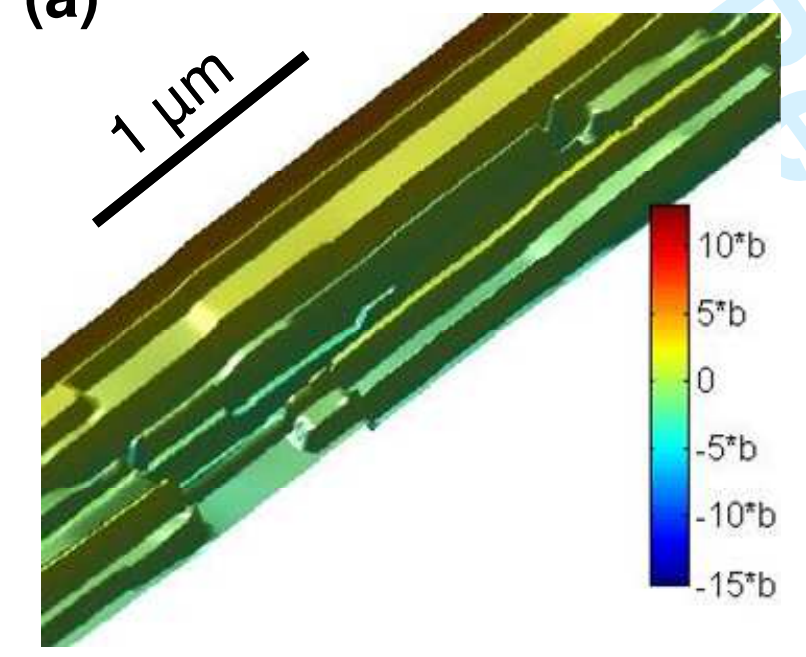

(c)

(b)

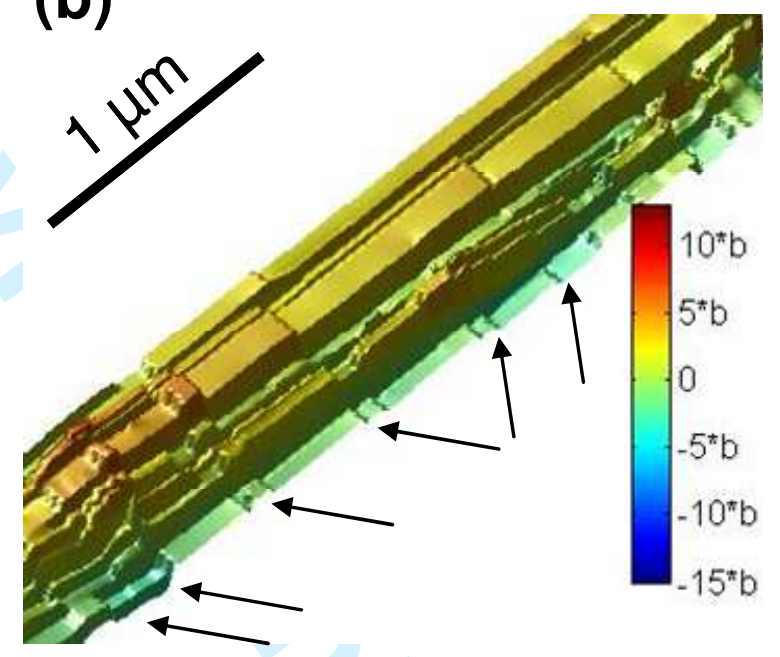

(d)

Figure-4. Results obtained in a simulation volume containing a mono-modal distribution of small particles. (a) Persistent slip band dislocation microstructure after $\mathrm{N}=3$ cycles, (b) threedimensional view of the dislocation microstructure after $\mathrm{N}=3$ cycles, (c) surface displacements associated with a persistent slip band, after $\mathrm{N}=1$ cycle, (d) after $\mathrm{N}=3$ cycles. 


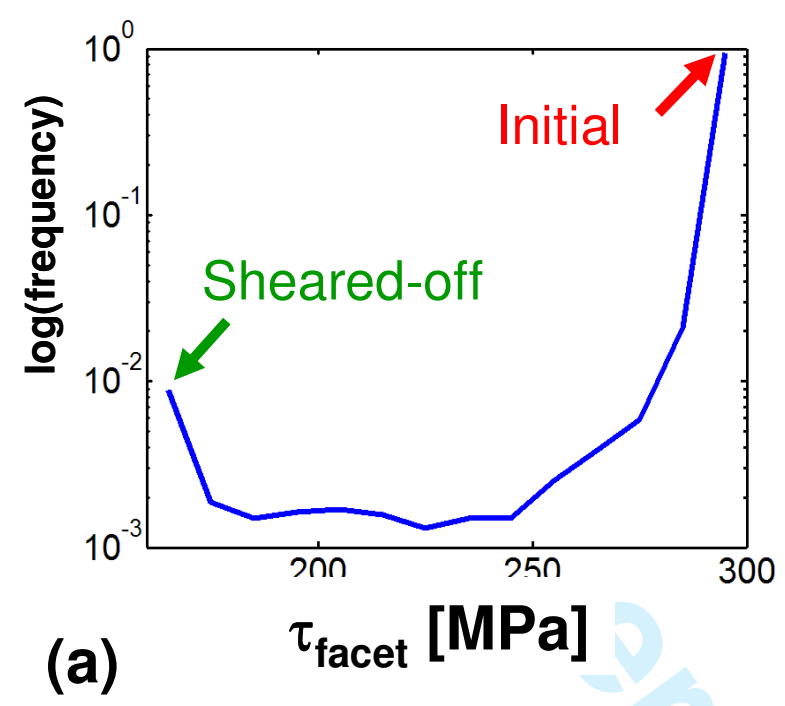

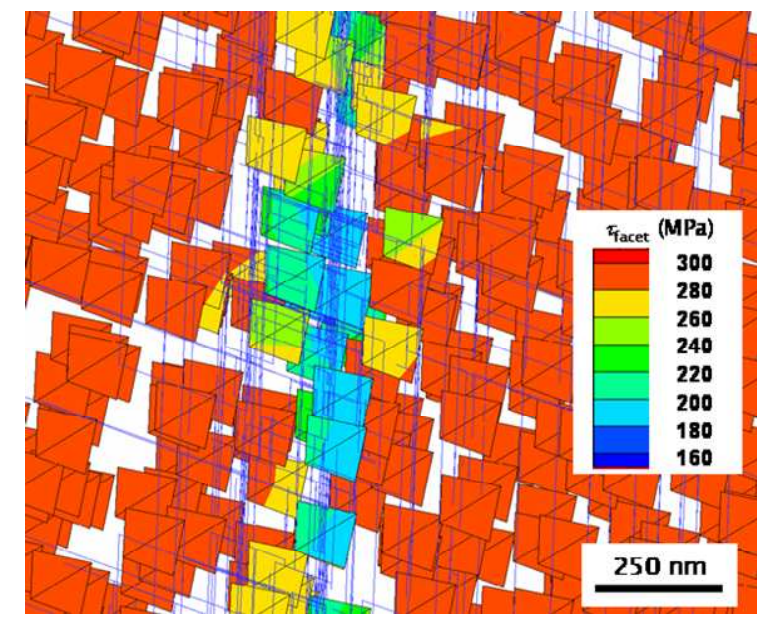

(b)

Figure-5. Results obtained in a simulation volume containing a mono-modal distribution of small particles. (a) Mapping of the residual mechanical strength of the particles, superimposed on the dislocation microstructure, (b) statistical analysis of the residual mechanical strength of the particles. 


\section{Figure-6}

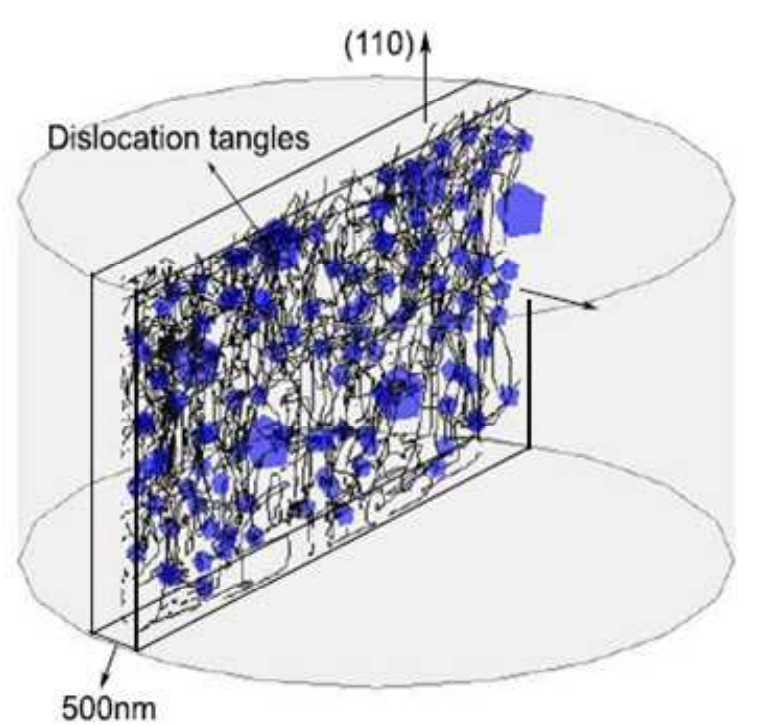

(a)
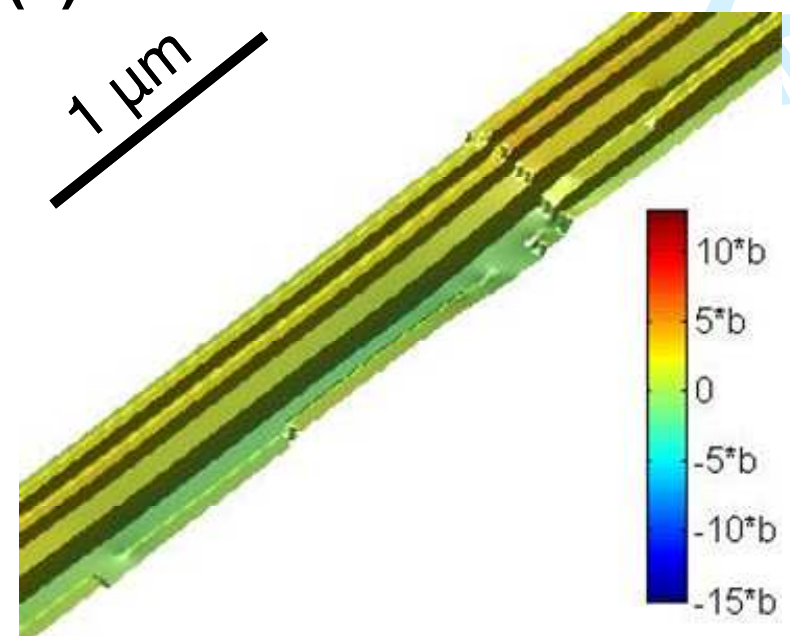

(c)

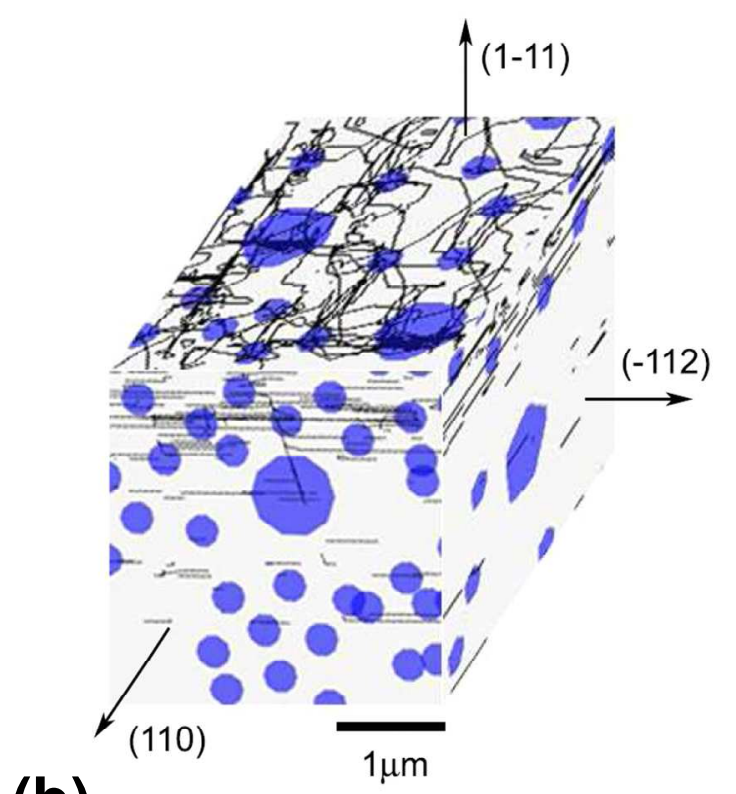

(b)

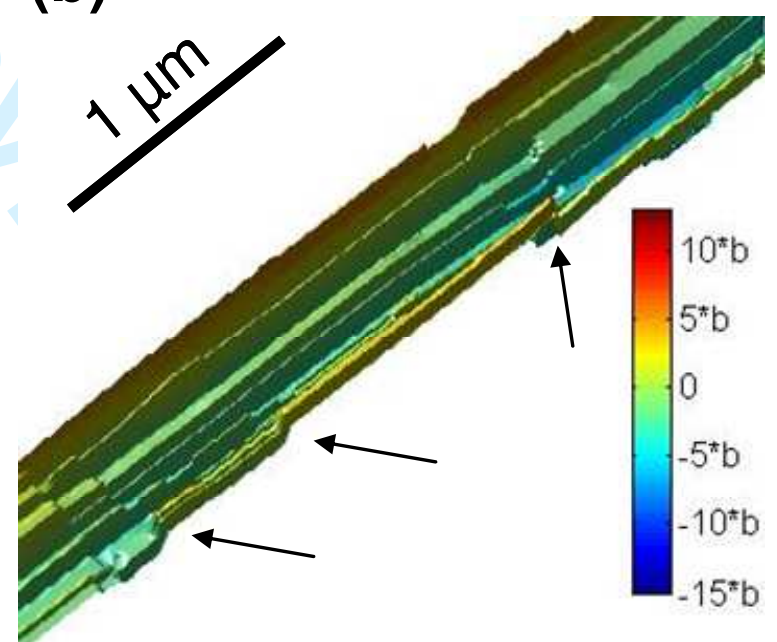

(d)

Figure-6. Results obtained in a simulation volume containing a bi-modal distribution of small and large particles. (a) Persistent slip band dislocation microstructure after $\mathrm{N}=4$ cycles, (b) three-dimensional view of the dislocation microstructure after $\mathrm{N}=4$ cycles, (c) surface displacements associated with a persistent slip band after $\mathrm{N}=1$ cycle, (d) after $\mathrm{N}=4$ cycles. 


\section{Figure-7}
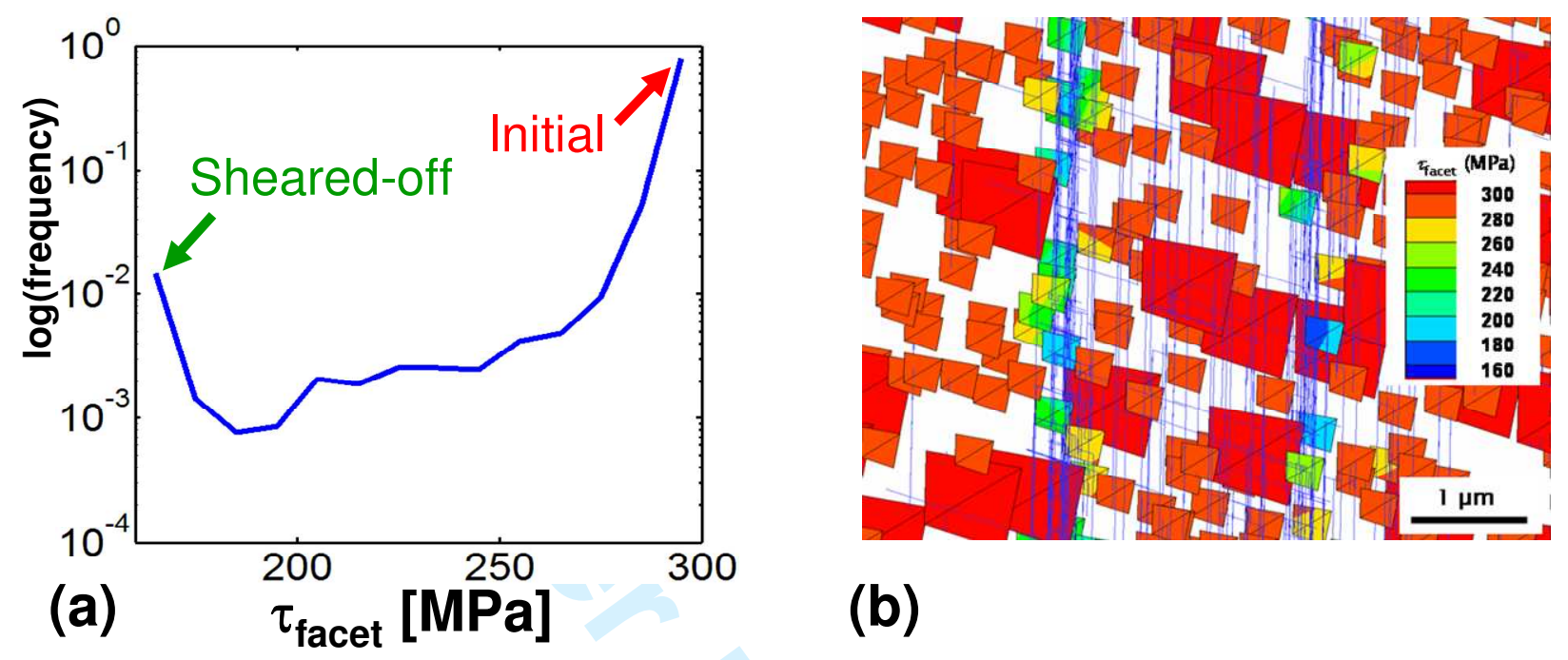

(b)

Figure-7. Results obtained in a simulation volume containing a bi-modal particle distribution. (a) statistical analysis of the residual mechanical strength of the particles, (b) mapping of the residual mechanical strength of the particles, superimposed on the dislocation microstructure. 